\title{
Samuel J. Klempner: approaching gastric cancer questions from different angles increases the chance of success
}

Submitted Jun 23, 2018. Accepted for publication Jun 26, 2018.

doi: $10.21037 /$ tcr.2018.06.23

View this article at: http://dx.doi.org/10.21037/tcr.2018.06.23

\section{Editor's note}

Gastric cancer, a leading cause of cancer mortality, is a heterogeneous disease that is predicted to claim over ten thousand lives in the United States in 2018, according to an American Cancer Society's estimate (1). Current treatment of gastric cancer is considered suboptimal as the cancer is commonly addressed in a uniform fashion regardless of the disease subtype. Traditional classifications such as the Lauren classification (2) and the World Health Organization classification (3) emerged with an aim to subdivide the disease and guide patient therapy, yet are of limited clinical utility. With the advent of high-throughput genomic technologies, gastric cancer can now be examined meticulously at a molecular level, which gives rise to the establishment of various gastric cancer molecular classifiers.

As the Director of GI Oncology and the Precision Medicine Program at The Angeles Clinic and Research Institute, Cedars-Sinai Medical Center, USA, Prof. Samuel J. Klempner has been putting sustained effort over the years in defining molecular subtypes in gastric and other gastrointestinal cancers, figuring out the mechanisms of resistance to targeted therapies, studying the intersection of genomics and immunotherapy, as well as carrying out early phase clinical trials. This time, we are honored to interview Prof. Klempner who will share with us the significance and limitations of the current gastric cancer molecular classifications, the mechanisms of acquired resistance to targeted therapies, some of his findings and interesting stories in his research.

\section{Expert introduction}

Samuel J. Klempner, MD (Figure 1) currently serves as the Director of GI Oncology and Precision Medicine at The Angeles Clinic and Research Institute, Samuel Oschin Comprehensive Cancer Institute, Cedars-Sinai Medical Center in the United States. He is a clinical and translational researcher focusing on experimental

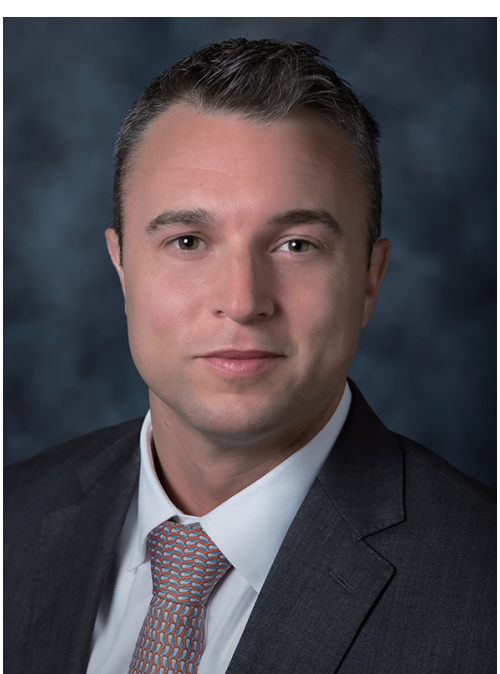

Figure 1 Prof. Samuel J. Klempner.

therapeutics in solid tumors, particularly gastrointestinal malignancies.

Prof. Klempner completed his residency in internal medicine at Brigham and Women's Hospital/Harvard Medical School, followed by a combined hematologyoncology fellowship at Beth Israel Deaconess Medical Center/Harvard Medical School. While at Harvard, he studied the mechanisms of resistance to targeted therapies in tumor cells in the lab of Dr. Lewis Cantley, PhD. He served as an Assistant Professor at the University of California Irvine (2013-2016) prior to joining The Angeles Clinic and Research Institute.

Prof. Klempner is currently an Editorial Board Member of Translational Cancer Research (TCR). His research has been presented at major international meetings including the American Society of Clinical Oncology (ASCO), European Society of Medical Oncology (ESMO), and the American Association for Cancer Research (AACR). Besides, he published in prestigious journals such as the Fournal of Clinical Oncology (JCO), Cancer Discovery, FAMA 
Oncology, Annals of Oncology and others. He is also active in SWOG and serves on several NIH committees.

\section{Interview}

TCR: Molecular classification of gastric cancer bas been prevalently studied in recent years. How will this assist clinical work in the long run?

Prof. Klempner: All cancers harbor some degree of genomic changes, and examination of genomic alterations is important for all tumor types. Pan-cancer analyses have the ability to identify recurrent patterns across anatomically and morphologically different tumor types to inform shared pathogenesis. Within gastric cancer advances in understanding the molecular landscape of both non-metastatic and advanced disease have added important knowledge to both biologic understanding and clinical care via identification of important prognostic and predictive biomarkers. Linking genomic changes to clinical data holds the promise of optimizing outcomes while simultaneously reducing potential toxicity in patients unlikely to benefit from a given therapy. Similarly, the incorporation of genomic data with current clinicopathologic variables in non-metastatic patients may ultimately risk-stratify patients for perioperative/ adjuvant strategies and surveillance. The bottom line is that overlaying genomic (and other -omic) data on clinical parameters will allow us to give our patients more granular estimates of both prognosis and chance of benefit from a given therapy.

\section{TCR: What are the limitations of the current classification system? How can it be further refined?}

Prof. Klempner: No classification system is without its limitations. The seminal The Cancer Genome Atlas (TCGA) and Asian Cancer Research Group (ACRG) gastric cancer molecular classification schema are no exception. The TCGA classification $(n=295)$ is almost exclusively derived from non-metastatic tumor samples; and extrapolation to metastatic patients cannot be definitively concluded. The ACRG classification is also largely nonmetastatic patients with a sample size of 300 . Computational studies have suggested at least 600 samples for a given anatomic tumor site to be optimal to fully characterize rare variants. Genomic heterogeneity underlies therapeutic resistance in many gastric cancer patients and the current classification schema are not designed to examine issues of baseline intra-tumoral and inter-tumoral heterogeneity. The ability to translate a classification schema into routine clinical practice requires several steps; And simplification of the current genomic categories will be important to increase adoption outside the research setting. Large collaborative efforts linking genomic events with outcomes across disease settings (essentially a much larger $\mathrm{n}$ ) is likely the best way to optimize current genomic classification. Similarly, incorporation of additional -omics platforms and ctDNA may refine the prognostic and predictive implications of a molecularly defined subset.

\section{TCR: Acquired resistance to targeted therapies remains a substantial challenge to clinical management of advanced cancers. What are the reasons behind?}

Prof. Klempner: Acquired resistance to targeted therapies is universal across all tumor types to varying degrees. Some truly more genomically homogenous tumors such as CML, GIST, and $A L K$-rearranged NSCLC can have very durable benefit from small molecule inhibitors, but in most other tumor types the benefit is less persistent owing to both innate and acquired resistance. Many researchers continue to study this important question, and some broad themes have emerged in acquired resistance to targeted therapies. Secondary alterations in the target that impact drug binding or degree of inhibition, the development of bypass tract activation, downregulation/loss of the target via genomic mechanisms, and outgrowth of a population initially lacking the target represent recurrent themes in acquired resistance. Tumors are incredibly adaptable to therapeutic stressors. Serial tumor biopsies and circulating tumor DNA (ctDNA) have been instrumental in elucidating mechanisms of acquired resistance across tumors. What is striking in gastric cancer is the baseline heterogeneity and impact of both intra- and inter-tumoral differences on duration of benefit from targeted therapies. We are looking at multi-region primary tumor biopsies and several recent papers from colleagues have highlighted heterogeneity and changes among Her2, MET, and EGFR during gastric cancer treatment. This poses a major problem for the field and novel combinatorial approaches with improved/ standardized assessment of heterogeneity will be important for targeted therapies in gastric cancer. 
TCR: Your research has been involved in identifying new therapies by studying the intersection of genomics and immunotherapy. What have been your findings so far?

Prof. Klempner: The intersection of genomic alterations and immune therapies has been known for some time and is highlighted by the association of lymphocyte infiltration and microsatellite instability (MSI) across tumor types. The ability to exploit this observation is relatively more recent and reflected in the durable responses seen with both PD-1 monotherapy and combination PD-1/CTLA-4 in MSI-high patients. We, and many others, are interested in exploring features of both responders and non-responders to immune checkpoint inhibitors, particularly with respect to genomic events. Certainly, there are going to be non-genomic mediators of response and resistance as well. For example, there is a clear relationship between tumor mutational burden (TMB) and response to immunotherapies in NSCLC, but less is known in GI cancers. We have noted that there are clear TMB-high/microsatellite stable subsets across GI cancers and are exploring the relationship with immunotherapy response. It is likely that different tumor types will have differing TMB thresholds distinguishing those more likely to respond from those less likely. We are also examining the genomic context among these patients as some innate resistance alterations may offset the potential predictive power of TMB. Characterizing exceptional responders can often provide biologic insights and is something we are examining in collaboration with others.

\section{TCR: What do you regard as the most difficult aspects of research? How do you deal with them?}

Prof. Klempner: The most difficult aspects of doing research are patience/time and balance between clinical duties and dedicated time to conduct research. Unfortunately, advanced gastric and esophageal cancers are frequently symptomatic diseases requiring a multidisciplinary team of subspecialists and time-intensive frequent care coordination. I have not yet figured how to add more hours to the day or phase out sleep, and like many others spend evenings trying to address research obligations. Often, we want things to come together quickly and fit the hypothesis one has generated; And trying to remain open and unbiased when examining your own data can be difficult which is why collaboration and peer review are critical. Many times, research questions just don't work out the way you imagine, though I do believe we can always learn something from the process. As nearly all clinical and basic researchers share similar stressors of time management and research funding I have found it very helpful to have informal meetings with colleagues to compare strategies and stories. Local and extramural mentorship cannot be understated in dealing with the difficulties of a clinical and translational research career.

\section{TCR: Your clinical work involves a wide range of areas. Which area is most interesting and most challenging to you?}

Prof. Klempner: As a GI medical oncologist, the large majority of my clinical work involves caring for patients with esophagogastric cancers. Uncommon oncogenic driver alterations and acquired resistance have always been an additional area of interest to me. Among the tumor types I see, gastric and esophageal cancers are my greatest interest, but also most challenging. I find both the clinical and genomic heterogeneity fascinating, but also frustrating as this aspect limits the efficacy of targeted therapies. There remain some relatively large understudied areas of gastric cancer, and I find ascites and peritoneal disease to be very interesting. I often wish gastric cancers more closely paralleled NSCLC as conceptualizing the paradigm of acquired resistance to a potent oncogenic driver (ALK, RET, EGFR, ROS1, etc.) is a framework that is easier for me to grasp. Having some experience with wiring, a signal transduction pathway is theoretically more analogous to the wiring diagram for a single-pole switch or circuit in series whereas gastric cancers are like multiple overlapping circuits in parallel.

\section{TCR: How does your clinical work assist your research, and vice versa?}

Prof. Klempner: The majority of my research occurs in the clinic and in collaboration with lab-based colleagues. Observations in the clinic drive many of the scientific questions we are interested in addressing. For example, we have seen several MSI-high patients fail to benefit from immunotherapies despite the very strong scientific rationale. Why is this? What is different about this patient or group of patients? How can we work with our basic science colleagues to address this observation? The communication between the clinic and lab is incredibly intellectually stimulating, and learning how to approach a question from many angles increases the chance of success. Clinical trial participation also fosters collaboration, and in early phase 
clinical trials aspects of the science are still unknown so there is the possibility to both offer patients a therapy that may benefit them while simultaneously helping to advance the basic understanding of the compound or pathway.

\section{TCR: As an editorial board member of TCR, would you say a few words to the peers in your field?}

Prof. Klempner: I would only re-iterate what my parents, boss on the farm, research and clinical mentors have imparted to me; never be satisfied and never stop asking why. Find a way to participate in research in some way as the only way we can improve outcomes is working together.

\section{Acknowledgments}

We would like to express our sincerest gratitude to Prof. Samuel J. Klempner for sharing his insights and opinions with us.

Funding: None.

\section{Footnote}

Provenance and Peer Review: This article was commissioned by the Editorial Office, Translational Cancer Research. The article did not undergo external peer review.

Conflicts of Interest: The author has completed the ICMJE uniform disclosure form (available at http://dx.doi. org/10.21037/tcr.2018.06.23). The author has no conflicts of interest to declare.

Ethical Statement: The author is accountable for all aspects of the work in ensuring that questions related to the accuracy or integrity of any part of the work are appropriately investigated and resolved.

Open Access Statement: This is an Open Access article distributed in accordance with the Creative Commons Attribution-NonCommercial-NoDerivs 4.0 International License (CC BY-NC-ND 4.0), which permits the noncommercial replication and distribution of the article with the strict proviso that no changes or edits are made and the original work is properly cited (including links to both the formal publication through the relevant DOI and the license). See: https://creativecommons.org/licenses/by-nc-nd/4.0/.

\section{References}

1. American Cancer Society [Internet]. The United States: American Cancer Society; 2018. Available online: https:// www.cancer.org/cancer/stomach-cancer/about/keystatistics.html

2. Laurén P. The two histological main types of gastric carcinoma: diffuse and so-called intestinal-type carcinoma. Acta Pathol Microbiol Scand 1965;64:31-49.

3. WHO Classification of Tumours of the Digestive System 4th eds. International Agency for Research on Cancer, 2010.

(Science Editor: Brad Li, TCR, tcr@amepc.org)
Cite this article as: $\mathrm{Li} \mathrm{B}$. Samuel J. Klempner: approaching gastric cancer questions from different angles increases the chance of success. Transl Cancer Res 2018;7(Suppl 7):S806S809. doi: 10.21037/tcr.2018.06.23 